\title{
Fucoidan attenuates hyperoxia-induced lung injury in newborn rats by mediating lung fibroblasts differentiate into myofibroblasts
}

\author{
Yan Zhang ${ }^{1 \#}$, Hengjian Du ${ }^{2 \#}$, Xuelian Yu ${ }^{1}$, Jiang $Z \mathrm{Zu}^{3}$ \\ ${ }^{1}$ Department of Geriatric Medicine, Sichuan Academy of Medical Sciences \& Sichuan Provincial People's Hospital, University Hospital of Electronic \\ Science \& Technology, Chengdu, China; ${ }^{2}$ Department of Geriatric Infectious Diseases, Sichuan Academy of Medical Sciences \& Sichuan Provincial \\ People's Hospital, University Hospital of Electronic Science \& Technology, Chengdu, China; ${ }^{3}$ Department of Respiratory and Critical Care \\ Medicine, Sichuan Academy of Medical Sciences \& Sichuan Provincial People’s Hospital, University Hospital of Electronic Science \& Technology, \\ Chengdu, China \\ Contributions: (I) Conception and design: Y Zhang, H Du, J Zhu; (II) Administrative support: J Zhu; (III) Provision of study materials or patients: \\ J Zhu, X Yu; (IV) Collection and assembly of data: Y Zhang, H Du, X Yu; (V) Data analysis and interpretation: Y Zhang, H Du; (VI) Manuscript \\ writing: All authors; (VII) Final approval of manuscript: All authors. \\ \#These authors contributed equally to this work. \\ Correspondence to: Jiang Zhu. Department of Respiratory and Critical Care Medicine, Sichuan Academy of Medical Sciences \& Sichuan Provincial \\ People's Hospital, University Hospital of Electronic Science \& Technology, No. 32 West Second Section, First Ring Road, Chengdu, China. \\ Email: nsjhg81757@sina.cn; Xuelian Yu. Department of Geriatric Medicine, Sichuan Academy of Medical Sciences \& Sichuan Provincial \\ People's Hospital, University Hospital of Electronic Science \& Technology, No. 32 West Second Section, First Ring Road, Chengdu, China. \\ Email: 2036623998@qq.com.
}

Background: Hyperoxia-induced lung injury is one of the most common and frequent diseases in premature infants and may develop into bronchopulmonary dysplasia (BPD). Fucoidan, extracted from brown seaweed and brown algae, has anti-apoptosis, antioxidative and anti-fibrosis effects. This study aimed to explore whether fucoidan could alleviate hyperoxia-induced lung injury in newborn rats.

Methods: Lung wet-weight/dry-weight (W/D) ratio, total protein (TP) content, total cell counts, and lactate dehydrogenase (LDH) levels are used to evaluate lung injury. Masson staining is used to evaluate lung fibrotic. Tunnel assay and Hoechst 33258 assay were used to evaluate apoptosis. The levels of serum superoxide dismutase (SOD), malondialdehyde (MDA), and glutathione (GSH) were measured using ELISA to assess oxidative stress. Western blot assay was used to detect apoptosis-related proteins Bcl-1, Bax, and myofibroblast proteins $\alpha$-SMA.

Results: The data indicating fucoidan treatment remarkably reduces the lung W/D ratio and TP content, total cell counts, and LDH levels in bronchoalveolar lavage fluid (BALF). Also, fucoidan treatment significantly inhibited cell apoptosis with the elevated expression of Bcl-2/Bax in cultured lung fibroblasts. Moreover, treatment with fucoidan suppressed the levels of MDA significantly and elevated the level of SOD and GSH, showing that oxidative stress was restrained by fucoidan. Furthermore, the decreased expression levels of $\alpha$-SMA and collagen I was detected in fibroblast treated with fucoidan.

Conclusions: These data suggest fucoidan may protect the lung from hyperoxia via suppressing cell apoptosis, mitigating oxidative stress, and inhibiting lung fibroblasts from differentiating into myofibroblasts.

Keywords: Fucoidan; fibrosis; apoptosis; hyperoxia-induced lung injury; bronchoalveolar lavage fluid (BALF)

Submitted Sep 03, 2020. Accepted for publication Nov 06, 2020.

doi: 10.21037/atm-20-6601

View this article at: http://dx.doi.org/10.21037/atm-20-6601 


\section{Introduction}

Hyperoxia is commonly encountered in premature infants with severe insufficient pulmonary functions (1). Highconcentration oxygen inhalation results in arrest in alveolarization and angiogenesis in the developing lung, which was called bronchopulmonary dysplasia (BPD) in the clinic (2). Although various therapeutic methods, including ventilation strategies, surfactant treatment, and prenatal steroids, have been making progress in reducing hyperoxia, the incidence of BPD remains high and often leads to longterm consequences of lung function (3). In recent years, new research findings provide new ideas for the treatment of BPD. The Nrf2/HO-1 axis plays important role in bronchopulmonary dysplasia and hyperoxic lung injuries (4). Heme oxygenase exerted positive role in neonatal lung injury and repair (5). Fucoidan exerted protective role in hyperoxia induced lung injury by regulating ERK signaling pathway (6). More treatments need to be explored. Thus, it is urgently needed to explore an effective therapeutic strategy that alleviates the adverse effects of hyperoxia on the developing lung.

Fucoidan, a class of complex L-fucose-enriched sulfated polysaccharides, is mainly found in brown seaweed and brown algae (7). According to published reports, fucoidan was widely used in various diseases, including type 2 diabetes-induced hindlimb, ischemic injury (8), hepatitis B (9), ethanol-induced gastric mucosal damage (10), and breast cancer (11). In these diseases, fucoidan exhibited potent anti-cancer, anti-coagulative, anti-inflammatory, anti-viral and antioxidative abilities $(12,13)$. Among these pharmacological properties, the anti-inflammatory and antioxidative effects of fucoidan may achieve the purpose of alleviating hyperoxia-induced lung injury in newborn rats. Nie et al. reported that fucoidan can play a protective role in hyperoxia induced lung injury by regulating ERK signaling pathway (6). However, little information is available on the effect of fucoidan on lung fibroblasts in lung injury induced by hyperoxia.

It is well-known that lung fibroblasts, important interstitial cells in lung tissues, play a vital role in normal and abnormal lung function and development (14). In the process of tissue injury and repair, lung fibroblasts would migrate into the provisional matrix, and then proliferate and produce the main components of the extracellular matrix (ECM), including fibronectin and collagen (15). Previous studies have indicated that hyperoxia would promote fibroblasts to differentiation into myofibroblasts in newborn rats (16). Myofibroblasts possess a more vital ability to synthesize collagen than normal fibroblasts. However, excessive collagen deposition would result in the steady deterioration of the fibrotic process, which plays a vital role in the pathogenetic process of BPD. Therefore, restraining fibroblasts that differentiate into myofibroblasts might be a potential target for the treatment of hyperoxia-induced acute lung injury.

In our research, we explore the possible protective effects and underlying molecular mechanisms of fucoidan on hyperoxia-induced acute lung injury in newborn rats. Results strongly showed that fucoidan treatment improved hyperoxia-induced acute lung injury through suppressing lung fibroblasts differentiate into myofibroblasts. We present the following article in accordance with the ARRIVE reporting checklist (available at http://dx.doi. org/10.21037/atm-20-6601).

\section{Methods}

\section{Animals and etbics}

Timed pregnant Sprague-Dawley rats (male, 7-8 weeks old, 220-240 g) were obtained from the Animal Center of Sichuan University. Pups were delivered naturally at full term and supported in an SPF environment with room air for two hours. Then, all pups are randomly divided into four groups (n=12 per group): control group, Fucoidan group, Hyperoxia group, and Hype + Fucoidan group. Fucoidan (HPLC $\geq 98 \%$ ) was purchased from Sigma-Aldrich (St. Louis, MO, USA) and dissolved in normal saline (NS) to $20 \mathrm{mg} / \mathrm{mL}$ for storage at $-20^{\circ} \mathrm{C}$. Rats in the control group and Fucoidan group were maintained in room air from postnatal day 1 (P1) to P22, and rats in the Hyperoxia group and Hype + Fucoidan group were housed in $80 \%$ oxygen conditions from $\mathrm{P} 1$ to $\mathrm{P} 22$. On P8, rats in the Fucoidan group and Hype + Fucoidan group were intraperitoneally injected with fucoidan $(80 \mathrm{mg} / \mathrm{kg} /$ day in NS), and other rats were intraperitoneally injected with NS in the same volume. The treatment continued for three days. On P22, pups were sacrificed by the administration of intraperitoneal pentobarbital $(50 \mathrm{mg} / \mathrm{kg})$. The Ankang Traditional Chinese Medicine Hospital laws approved all animal protocols governing animal care (No. SCAMS190321).

\section{Lung wet weight and dry weight}

For the measurement of pulmonary edema, the lung tissues were weighed at once after separated from sacrificed rats. Then, tissues were placed in an oven for at $60{ }^{\circ} \mathrm{C}$ for 
48 hours. After measuring the dried lung tissue, tissue edema of male albino rats was examined by determination of the lung wet-weight/dry-weight ratio (W/D).

\section{Collection and analysis of bronchoalveolar lavage fluid (BALF)}

On P22, the pups were sacrificed. Then, rats were received tracheal intubation and injection of ice-cold $0.9 \%$ saline $(0.035 \mathrm{~mL} / \mathrm{g}$ body weight) for five times. BALF was collected, and the total volume was recorded. BALF was centrifuged $\left(400 \times \mathrm{g}, 15\right.$ minutes, $\left.4^{\circ} \mathrm{C}\right)$, and the cell pellets were resuspended in $200 \mu \mathrm{L}$ PBS (Gibco, Rockville, MD, USA). Total cells are quantified using a hemocytometer by the trypan blue exclusion method. Total protein (TP) was detected using Lowry assays. Lactate dehydrogenase (LDH) level was measured using an LDH assay kit (Nanjing Jiancheng Bioengineering Institute, Nanjing, China).

\section{Masson staining}

The isolated lung tissues were fixed with $10 \%$ formalin, embedded in paraffin, and cut into $4 \mu \mathrm{m}$ sections. Then, sections were subjected to Masson staining. Briefly, sections were incubated with hematoxylin for 6 minutes and then incubated with Ponceau-Acid Fuchsin solution for 1 minute. Subsequently, sections were washed with $0.2 \%$ glacial acetic acid and incubated with $1 \%$ phosphomolybdic acid for 5 minutes. The collagen fibers were stained with aniline blue. After dehydration, sections were observed under an inverted microscope (Olympus, Tokyo, Japan).

\section{TUNEL assay}

The previously described paraffin sections were deparaffinized and rehydrated. Then, sections were incubated with the TUNEL reaction mixture (Roche Applied Science, Penzberg, Germany) according to the instructions of the manufacturer. The positive cells were observed under a light microscope (Olympus).

\section{Elisa assays}

The levels of SOD, MDA, and GSH in serum were detected using ELISA kits (Dakewe, Shenzhen, China). The experiment was performed according to the manufacturer's instructions strictly. Optical absorption was assessed at $450 \mathrm{~nm}$ in a microplate reader (Bio-Rad Laboratories, Inc.,
Hercules, CA, USA).

\section{Primary lung fibroblast culture}

On P22, lung tissues isolated from each group were minced into $1-\mathrm{mm}^{3}$ pieces and dissociated in HBSS (Gibco) containing DNAse $(20 \mu \mathrm{g} / \mathrm{mL})$ and $0.25 \%$ trypsin at $37{ }^{\circ} \mathrm{C}$ for 15 minutes. Dissociation was stopped by adding DMEM (Gibco) with 10\% fetal bovine serum (FBS). The cells were filtered and then centrifuged at $650 \times \mathrm{g}$ for 10 minutes at $4{ }^{\circ} \mathrm{C}$. After that, cells were cultured with DMEM holding $10 \%$ FBS at $37^{\circ} \mathrm{C}$ with $5 \% \mathrm{CO}_{2}$.

\section{Hoechst 33258 assay for apoptosis}

Lung fibroblasts were seeded into a 12 -well plate. After cell adherence, cells isolated from each group received their treatment $(16 \mu \mathrm{g} / \mathrm{mL}$ fucoidan or the same volume of PBS) for 24 hours. Then, the cell apoptosis was measured by using Hoechst 33258 staining (Beyotime Institute of Biotechnology, Shanghai, China). Briefly, cells were fixed with methanol for 30 minutes. After washing with PBS, cells were incubated with Hoechst 33258 dye at room temperature for 10 minutes. Apoptotic cells were observed under a fluorescence microscope (Olympus).

\section{Western-blot assay}

Primary lung fibroblasts were seeded into a 6-well plate and treated as before. Then, cells were homogenized in the RIPA buffer, holding 1\% PMSF on the ice for 30 minutes. After centrifugation at $12,000 \times \mathrm{g}$ for 15 minutes, the supernatant was collected, and protein concentration was detected using BCA protein assay reagent (Solarbio, Beijing, China). Afterward, $10 \%$ SDS-PAGE separated proteins and transferred to PVDF membranes (Millipore, Billerica, MA). Membranes were blocked with $5 \%$ non-fat milk for 1.5 hours. After washing, proteins were incubated with anti-Bcl-2, anti-Bax, anti- $\alpha$ SMA, and anti-Collagen I (Abcam, Cambridge, UK) overnight at $4{ }^{\circ} \mathrm{C}$. After washing with TBST, these membranes were then incubated with horseradish peroxidase-labeled antibody (Abcam) for 1 hour at room temperature. Immunoreactive bands were measured using ECL reagents.

\section{RT-PCR assay}

After different treatment, total RNA was isolated from the primary lung fibroblast using Trizol Reagent (Sigma, 
Table 1 Primer sequences

\begin{tabular}{lll}
\hline Gene & Forward primer 5'-3' & Reverse primer 5'-3' \\
\hline$\alpha$-SMA & 5'-TGACGCTGAAGTATCCGATAGA-3' & 5'-CGAAGCTCGTTATAGAAAGAGTGG-3' \\
Collagen I & 5'-AGATAAGGG TCCAACTGGTGT-3' & 5'-ACCTTTAACGGCACCTAAAATGA-3' \\
U6 & 5'-GCTTCGGCAGCACATATACTAAAAT-3' & 5'-CGCTTCACGAATTGCGTGTCAT-3' \\
\hline
\end{tabular}

St. Louis, MO, USA) according to the manufacturer's instructions. Then, RNA was reverse transcribed into cDNA using a reverse transcription reaction system (Takara Bio Inc., Dalian, China). RT-qPCR was performed with the SYBR RT-PCR kit (Takara Bio Inc.). The primer sequences are shown in Table 1.

\section{Statistical analysis}

Data are mean \pm SD. Multiple comparisons of parametric data were measured by one-way ANOVA, followed by Bonferroni multiple tests between group pairs (SPSS 17.0, SPSS, Chicago, IL). $\mathrm{P}<0.05$ was considered statistically significant.

\section{Results}

Fucoidan treatment alleviates hyperoxia-induced lung injury

The structure of fucoidan was illustrated in Figure $1 \mathrm{~A}$. Hyperoxia-exposed newborn rats were treated with fucoidan to explore the effect of fucoidan on hyperoxiainduced lung injury. As shown in Figure 1B, fucoidan treatment remarkably decreased the elevated lung W/D weight ratio induced by hyperoxia. Besides, the TP content, total cell counts, and LDH level of BALF were significantly reduced in the Hype + Fucoidan group compared with the Hyperoxia group (Figure 1C,D,E). These results showed that fucoidan treatment could remarkably attenuate hyperoxia-induced lung injury.

\section{Fucoidan treatment reduces the synthesis of collagen in byperoxia-exposed lung tissues}

Excessive collagen deposition would lead to a steady deterioration of the fibrotic process. Our study showed that hyperoxia resulted in collagen protein synthesis disorder and excessive deposition. However, fucoidan treatment returned the collagen protein content to the level of control rats (Figure 2).

Fucoidan therapy decreases cell apoptosis in hyperoxiaexposed lung tissues and cultured primary lung fibroblast

Hyperoxia-exposed rats and cultured primary lung fibroblasts were treated with fucoidan to detect whether fucoidan treatment could reduce cell apoptosis. As illustrated in Figure 3A, TUNEL assay suggested hyperoxiainduced cell apoptosis in lung tissues was remarkably suppressed by fucoidan treatment. Besides, the apoptosis of cultured lung fibroblasts was significantly decreased in the Hype + Fucoidan group compared with the Hype group (Figure 3B). Moreover, the Bcl-2/Bax ratio was elevated in the Hype + Fucoidan group compared with the Hype group (Figure 3C). These results strongly showed that fucoidan could tremendously reduce cell apoptosis in hyperoxiaexposed lung tissues and cultured primary lung fibroblasts.

\section{Fucoidan restrains oxidative stress in hyperoxia-exposed newborn rats}

To explore whether fucoidan treatment could alleviate oxidation reactions, the level of serum SOD, MDA, and GSH was measured in hyperoxia-exposed newborn rats using ELISA. Results suggested that SOD and GSH level was significantly elevated in Hype + Fucoidan group compared with Hype group (Figure $4 A, B, C$ ). In addition to this, hyperoxia-induced, the increase in the level of MDA was remarkably repressed by fucoidan therapy (Figure $4 B$ ). These results suggested that oxidative stress induced by hyperoxia could be remarkably improved by fucoidan treatment.

\section{Fucoidan suppresses lung fibroblasts that differentiate into myofibroblasts}

Myofibroblasts could express $\alpha$-SMA, and $\alpha$-SMA was related to the activation of myofibroblasts, which has a more significant effect on collagen production than 
A

Fucus vesiculosus<smiles>COC1C(C)OC(C)C(OC)C1OS(=O)(=O)O</smiles>

Fucoidan

$(\mathrm{C} 12 \mathrm{H} 18 \mathrm{CaO} 14 \mathrm{~S} 2) \mathrm{n}=(\mathbf{4 9 0 . 4 8 )}$
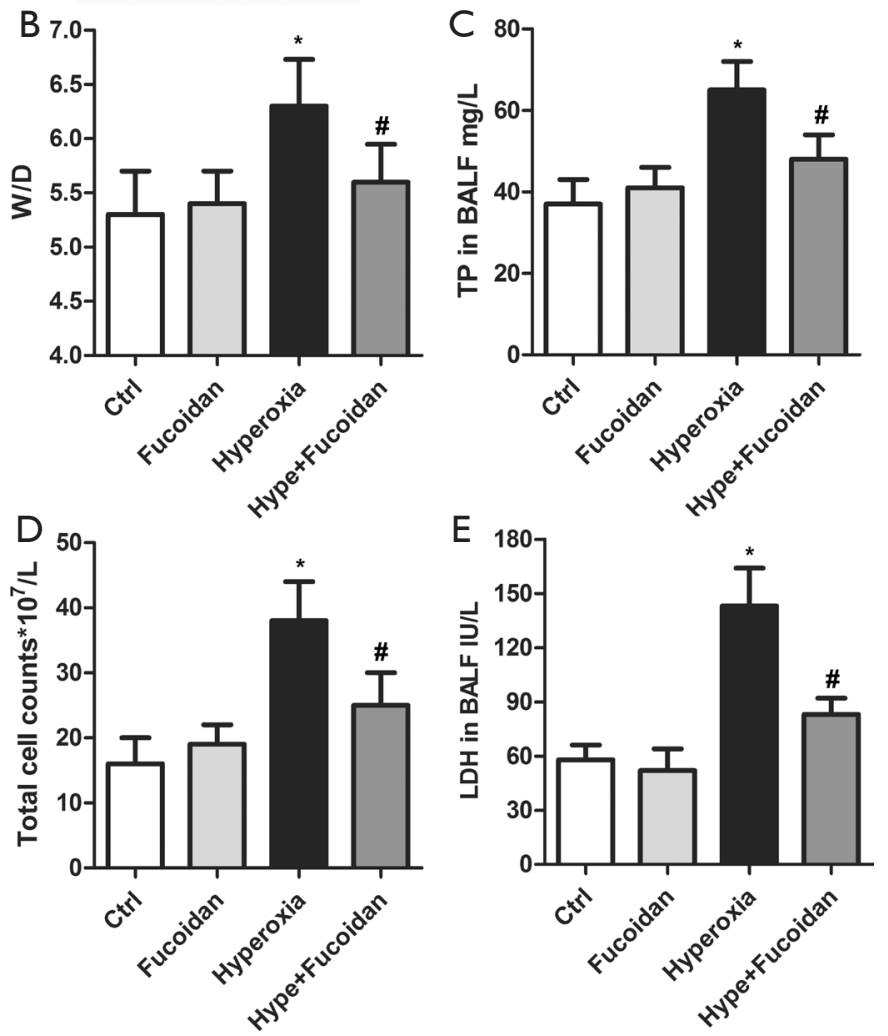

Figure 1 Fucoidan treatment alleviates hyperoxia-induced lung injury. (A) The structure of fucoidan. The effect of fucoidan on (B) lung wet/ dry weight ratio, and (C) TP content, (D) total cell counts, and (E) LDH in BALF. The experiments were repeated at least three times, and error bars represent \pm SD. *, $\mathrm{P}<0.05$ versus control group; *, $\mathrm{P}<0.05$ versus hyperoxia group. TP, total protein; LDH, lactate dehydrogenase; BALF, bronchoalveolar lavage fluid.
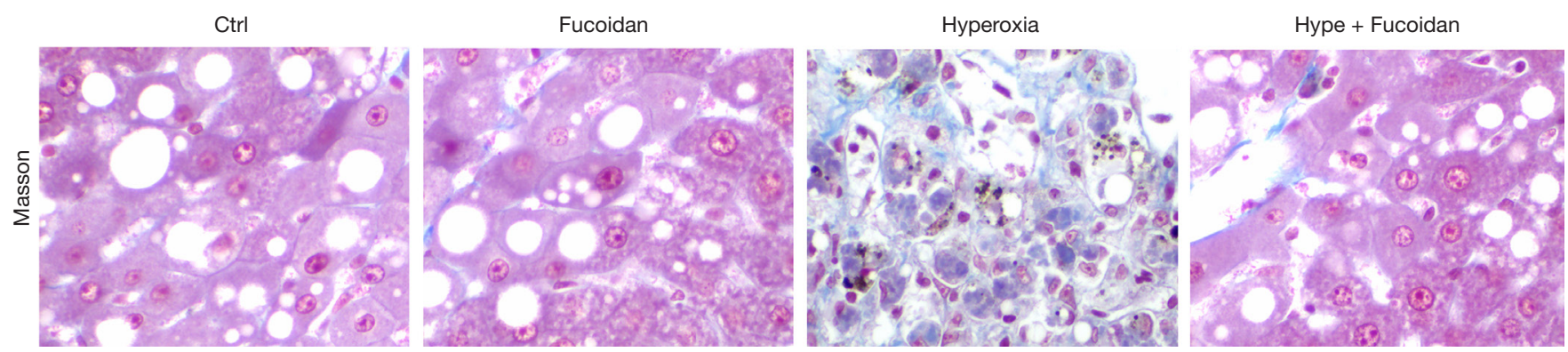

Figure 2 Fucoidan treatment reduces the synthesis of collagen in hyperoxia-exposed lung tissues. Masson staining was used to measure the expression level of collagen $(\times 200)$. 
A
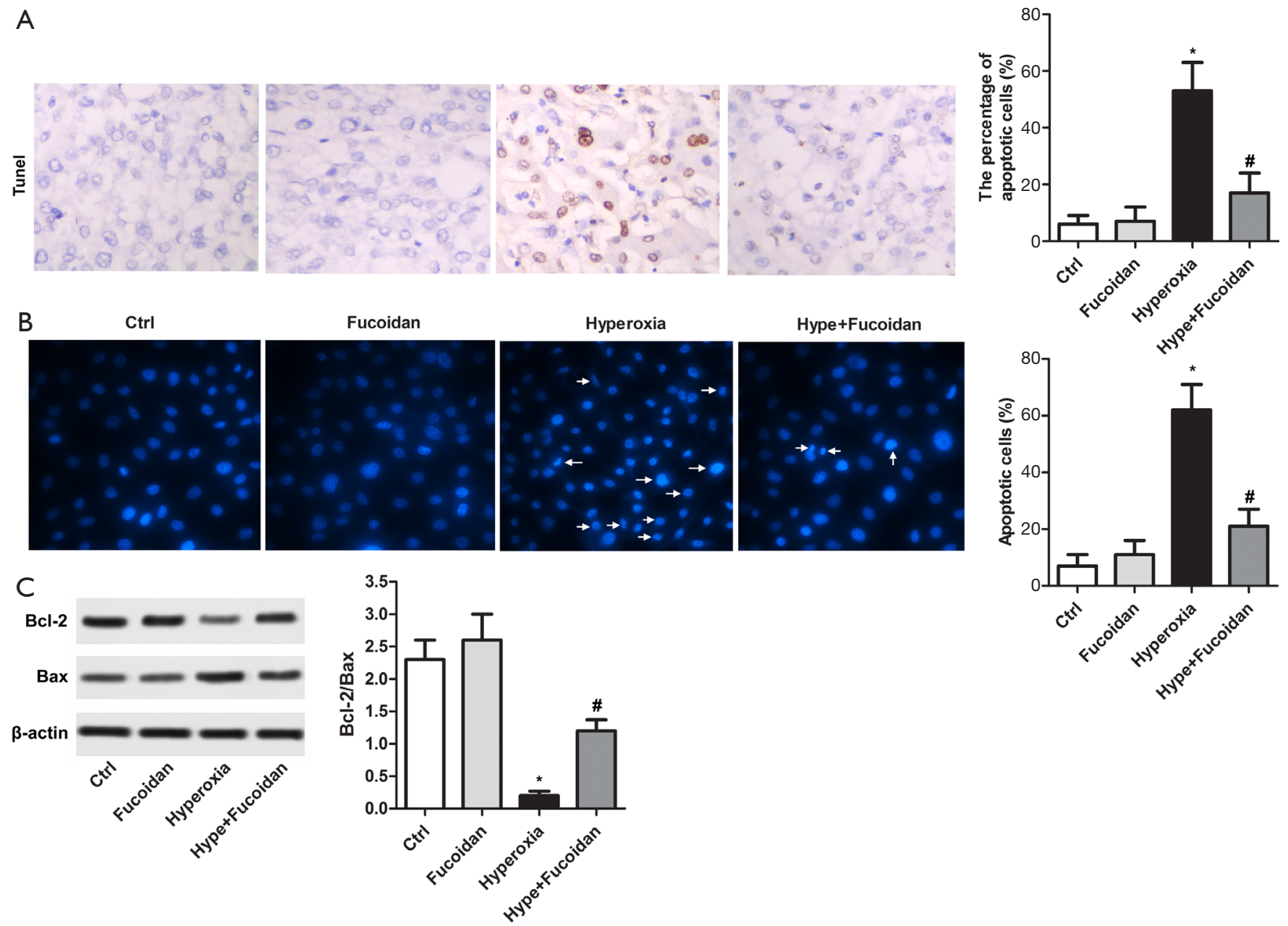

Figure 3 Fucoidan therapy decreases cell apoptosis in hyperoxia-exposed lung tissues and cultured primary lung fibroblast. (A) TUNEL staining was applied to assess cell apoptosis in lung tissues $(\times 200)$; (B) the apoptosis of cultured primary lung fibroblasts was evaluated using Hoechst staining $(\times 200)$. The arrow points to apoptotic cells; (C) the expression of Bcl-2/Bax was measured using western-blot. The experiments were repeated at least three times, and error bars represent $\pm \mathrm{SD}^{*}, \mathrm{P}<0.05$ versus control group; ${ }^{*}, \mathrm{P}<0.05$ versus the hyperoxia group. TUNEL, TdT-mediated DUTP nick end labeling.

normal fibroblasts. Results showed that hyperoxia-induced the increase in the protein and mRNA levels of $\alpha$-SMA and collagen I were significantly suppressed by fucoidan treatment (Figure $5 A, B, C$ ). These results suggested that fucoidan could inhibit lung fibroblasts from differentiating into myofibroblasts.

\section{Discussion}

The regeneration of reactive oxygen species due to hyperoxia incitement would elicit lung injury $(17,18)$. Studies have linked hyperoxia exposure to the development of BPD, one of the most common risk factors for mortality and morbidity in preterm neonates, and always follows ventilation and oxygen therapy for acute respiratory failure after premature birth (19). Currently, there is no effective therapeutic schedule for BPD. Therefore, preventing hyperoxia-induced lung injury from getting into BPD becomes an imperative requirement.

Fucoidan has attracted the attention of various researchers for its diverse biological activities. Thus, fucoidan has a highly considerable potential application in various fields, including food and drug (20). Besides, fucoidan has been reported to play a positive role in lung 

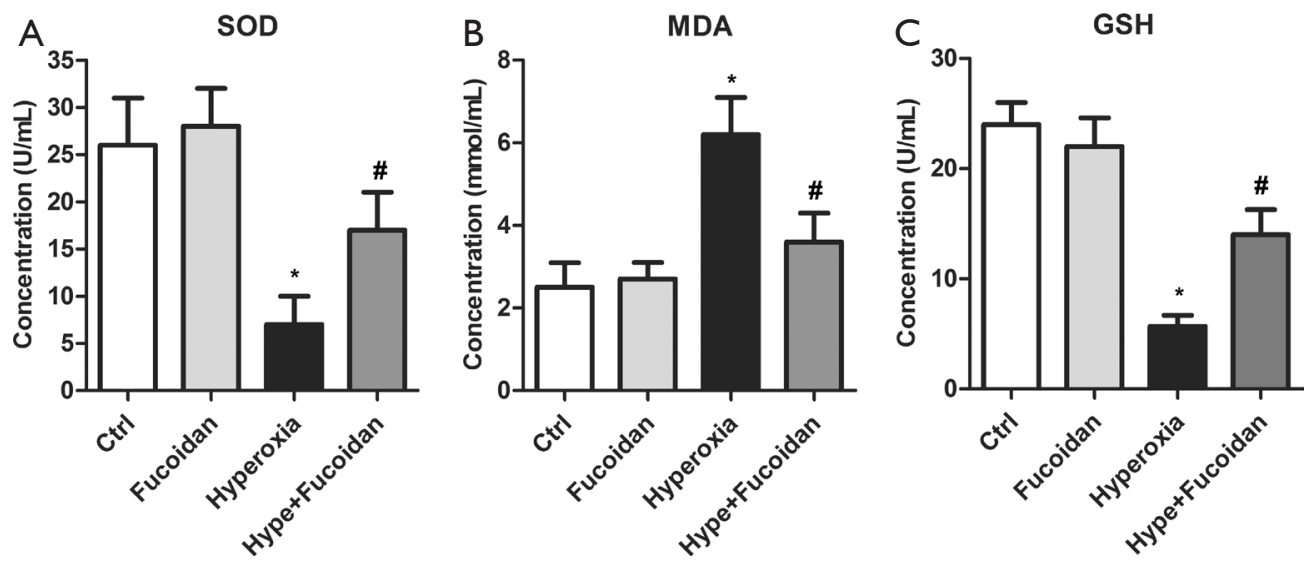

Figure 4 Fucoidan restrains oxidative stress in hyperoxia-exposed newborn rats. The level of (A) SOD, (B) MDA, (C) GSH was measured using ELISA. The experiments were repeated at least three times, and error bars represent $\pm \mathrm{SD} .{ }^{*}, \mathrm{P}<0.05$ versus control group; ${ }^{*}, \mathrm{P}<0.05$ versus the hyperoxia group. SOD, superoxide dismutase; MDA, malondialdehyde; GSH, glutathione.

A

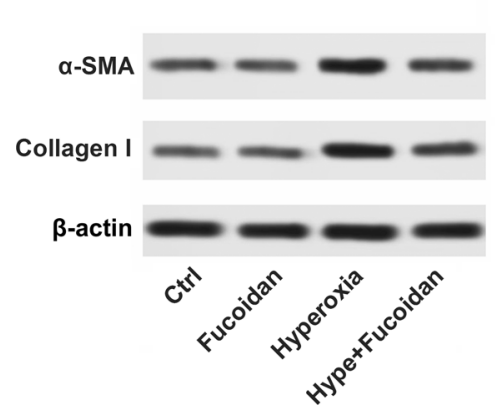

B

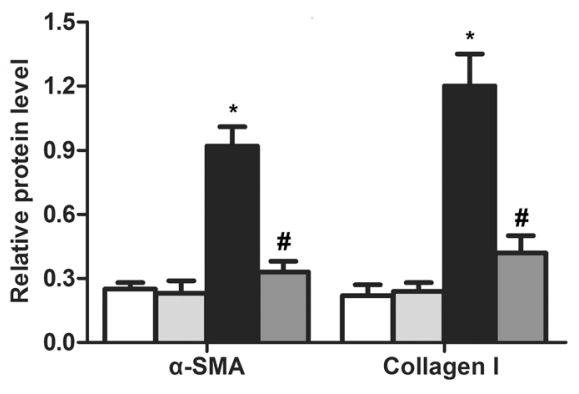

C
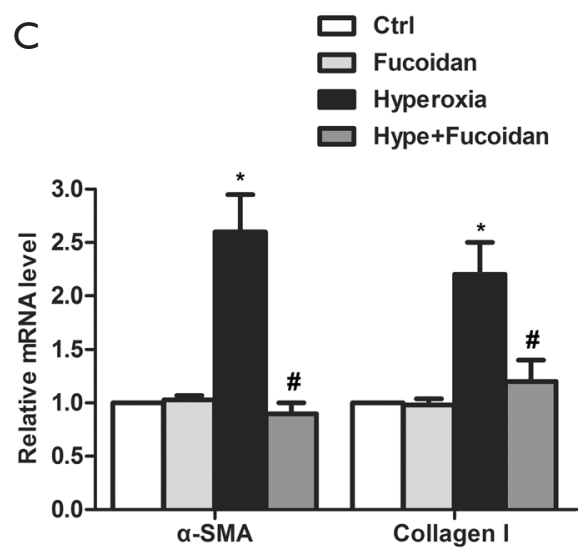

Figure 5 Fucoidan suppresses lung fibroblasts that differentiate into myofibroblasts. (A) The protein level of $\alpha$-SMA and collagen I was evaluated using western-blot; (B) relative protein level of $\alpha$-SMA and collagen I; (C) RT-PCR was used to detect the mRNA level of $\alpha$-SMA and Collagen I. The experiments were repeated at least three times, and error bars are $\pm \mathrm{SD}$. * $\mathrm{P}<0.05$ versus control group; ${ }^{*}, \mathrm{P}<0.05$ versus hyperoxia group. $\alpha$-SMA, alpha-smooth muscle actin.

injury induced by various pathogenic factors. According to Karzai et al., fucoidan reduced lung neutrophils, and these were significant with high tidal volumes (21). Besides, other researchers pointed out that systemic pretreatment with fucoidan suppressed platelet rolling, adherence, and the decrease in diameter, thereby inhibiting the development of pulmonary IR injury (22). Moreover, Nie and colleagues have demonstrated that atomization inhalation of fucoidan may ameliorate hyperoxic lung injury though modulating the ERK1/2 signaling pathway (6). Similarly, in our research, fucoidan treatment tremendously suppresses the lung W/D weight ratio and TP content, total cell count, and LDH level in BALF.

Cell apoptosis is one of the most common manifestations of lung injury induced by hyperoxia (23). Reducing apoptosis is of significant importance for the treatment of hyperoxia-induced acute lung injury. Many researchers have shown the anti-apoptosis effect of fucoidan on various cells. For example, fucoidan treatment statistically decreased the expressions of p-p53 and Bax and remarkably enhanced the levels of Bcl-2 in cerebral ischemia-reperfusion injury, indicating cell apoptosis was suppressed by fucoidan (24). Also, fucoidan treatment vastly alleviated the elevated pancreatic $\beta$-cell apoptosis and reduced insulin secretion 
in STZ-treated NIT-1 cells and mice (25). Moreover, low molecular weight fucoidan inhibits apoptosis and inflammation to protect against albumin overload, causing renal injury in chronic kidney disease (26). A similar result was drawn in our research; increased cell apoptosis induced by hyperoxia was remarkably suppressed by fucoidan, and the increased Bcl-2/bax accompanies the action.

The lung of a premature baby is not yet mature, so continuous inhalation of high concentrated oxygen may lead to oxidative stress injury in the lung tissue (27). The results obtained from the current study showed that fucoidan could attenuate oxidative stress in various diseases. According to Heeba et al., fucoidan mitigated steatohepatitis and insulin resistance through restraining oxidative stress and inflammatory cytokines in experimental non-alcoholic fatty liver disease (28). Besides, other researchers have indicated fucoidan defends mesenchymal stem cells against oxidative stress and elevates the regeneration of vascular in a murine hindlimb ischemia model (29). Furthermore, fucoidan was demonstrated to possess anti-dyslipidemic and antiatherosclerotic effects via inducing plasma lipoprotein lipase activity and suppressing the effects of inflammation and oxidative stress in HFD-fed ApoEshl mice (30). Similarly, in our research, oxidative stress was remarkably inhibited by fucoidan treatment, which was reflected by the increased expression of SOD and GSH along with the decreased MDA.

Pulmonary fibrosis is a vital process in lung injury induced by hyperoxia (31). Accumulated studies have shown that fucoidan has a potent anti-fibrosis effect on various diseases. It has been suggested and supported by studies fucoidan suppressed liver fibrosis via restraining the activation of hepatic stellate cells and the formation of ECM (32). Besides, Chen et al. observed oligo-fucoidan doses less than $100 \mathrm{mg} / \mathrm{kg} /$ day improved renal function and suppressed renal tubulointerstitial fibrosis in chronic kidney disease mice (33). Moreover, low-molecular-weight fucoidan could alleviate renal fibrosis via inactivating, transforming growth factor- $\beta$ in diabetic nephropathy in vivo and in vitro (34). Similarly, in our study, fucoidan treatment may remarkably attenuate lung fibrosis through inhibiting lung fibroblasts differentiate into myofibroblasts, which are manifested by the decreased expression levels of $\alpha$-SMA and collagen I.

\section{Conclusions}

In the present study, the results showed that fucoidan treatment might mitigate hyperoxia-induced lung injury via reducing cell apoptosis, alleviating oxidative stress, and inhibiting lung fibroblasts from differentiating into myofibroblasts. The present study suggested that fucoidan may be developed as a candidate drug for the treatment of hyperoxia-induced acute lung injury in newborn rats.

\section{Acknowledgments}

Funding: None.

\section{Footnote}

Reporting Checklist: The authors have completed the ARRIVE reporting checklist. Available at http://dx.doi. org/10.21037/atm-20-6601

Data Sharing Statement: Available at http://dx.doi. org/10.21037/atm-20-6601

Conflicts of Interest: All authors have completed the ICMJE uniform disclosure form (available at http://dx.doi. org/10.21037/atm-20-6601). The authors have no conflicts of interest to declare.

Ethical Statement: The authors are accountable for all aspects of the work in ensuring that questions related to the accuracy or integrity of any part of the work are appropriately investigated and resolved. The Ankang Traditional Chinese Medicine Hospital laws approved all animal protocols governing animal care (No. SCAMS190321).

Open Access Statement: This is an Open Access article distributed in accordance with the Creative Commons Attribution-NonCommercial-NoDerivs 4.0 International License (CC BY-NC-ND 4.0), which permits the noncommercial replication and distribution of the article with the strict proviso that no changes or edits are made and the original work is properly cited (including links to both the formal publication through the relevant DOI and the license). See: https://creativecommons.org/licenses/by-nc-nd/4.0/.

\section{References}

1. Zhang X, Lu A, Li Z, et al. Exosomes secreted by endothelial progenitor cells improve the bioactivity of pulmonary microvascular endothelial cells exposed to 
hyperoxia in vitro. Ann Transl Med 2019;7:254.

2. Bhandari A, Panitch HB. Pulmonary outcomes in bronchopulmonary dysplasia. Semin Perinatol 2006;30:219-26.

3. Kair LR, Leonard DT, Anderson JM. Bronchopulmonary dysplasia. Pediatr Rev 2012;33:255-63; quiz 263-4.

4. Amata E, Pittalà V, Marrazzo A, et al. Role of the Nrf2/ HO-1 axis in bronchopulmonary dysplasia and hyperoxic lung injuries. Clin Sci (Lond) 2017;131:1701-12.

5. Dennery PA. Heme oxygenase in neonatal lung injury and repair. Antioxid Redox Signal 2014;21:1881-92.

6. Nie M, Wang Y, Lu Y, et al. Protective effects of fucoidan against hyperoxic lung injury via the ERK signaling pathway. Mol Med Rep 2018;17:1813-8.

7. Ale MT, Mikkelsen JD, Meyer AS. Important determinants for fucoidan bioactivity: a critical review of structurefunction relations and extraction methods for fucosecontaining sulfated polysaccharides from brown seaweeds. Mar Drugs 2011;9:2106-30.

8. Wang Z, Liu T, Chen X, et al. Low molecular weight fucoidan ameliorates hindlimb ischemic injury in type 2 diabetic rats. J Ethnopharmacol 2018;210:434-42.

9. Li H, Li J, Tang Y, et al. Fucoidan from Fucus vesiculosus suppresses hepatitis B virus replication by enhancing extracellular signal-regulated Kinase activation. Virol J 2017;14:178.

10. Kan J, Hood M, Burns C, et al. A Novel Combination of Wheat Peptides and Fucoidan Attenuates Ethanol-Induced Gastric Mucosal Damage through Anti-Oxidant, AntiInflammatory, and Pro-Survival Mechanisms. Nutrients 2017;9:978.

11. Xue M, Ji X, Xue C, et al. Caspase-dependent and caspaseindependent induction of apoptosis in breast cancer by fucoidan via the PI3K/AKT/GSK3 $\beta$ pathway in vivo and in vitro. Biomed Pharmacother 2017;94:898-908.

12. Koyanagi S, Tanigawa N, Nakagawa H, et al. Oversulfation of fucoidan enhances its anti-angiogenic and antitumor activities. Biochem Pharmacol 2003;65:173-9.

13. Wang J, Zhang Q, Zhang Z, et al. Potential antioxidant and anticoagulant capacity of low molecular weight fucoidan fractions extracted from Laminaria japonica. Int J Biol Macromol 2010;46:6-12.

14. McGowan SE, Torday JS. The pulmonary lipofibroblast (lipid interstitial cell) and its contributions to alveolar development. Annu Rev Physiol 1997;59:43-62.

15. Fries KM, Blieden T, Looney RJ, et al. Evidence of fibroblast heterogeneity and the role of fibroblast subpopulations in fibrosis. Clin Immunol Immunopathol
1994;72:283-92.

16. Wang Y, Yue S, Luo Z, et al. N-methyl-D-aspartate receptor activation mediates lung fibroblast proliferation and differentiation in hyperoxia-induced chronic lung disease in newborn rats. Respir Res 2016;17:136.

17. Moorthy B, Parker KM, Smith CV, et al. Potentiation of oxygen-induced lung injury in rats by the mechanismbased cytochrome P-450 inhibitor, 1-aminobenzotriazole. J Pharmacol Exp Ther 2000;292:553-60.

18. Yang F, Coalson JJ, Bobb HH, et al. Resistance of hypotransferrinemic mice to hyperoxia-induced lung injury. Am J Physiol 1999;277:L1214-23.

19. Kiren V, Barbi E, Ventura A. Chronic lung disease after premature birth. N Engl J Med 2008;358:745; author reply -6 .

20. Wijesinghe WA, Jeon YJ. Exploiting biological activities of brown seaweed Ecklonia cava for potential industrial applications: a review. Int J Food Sci Nutr 2012;63:225-35.

21. Karzai W, Cui X, Heinicke N, et al. Neutrophil stimulation with granulocyte colony-stimulating factor worsens ventilator-induced lung injury and mortality in rats. Anesthesiology 2005;103:996-1005.

22. Roberts AM, Ovechkin AV, Mowbray JG, et al. Effects of pulmonary ischemia-reperfusion on platelet adhesion in subpleural arterioles in rabbits. Microvasc Res 2004;67:29-37.

23. Bao XC, Fang YQ, You P, et al. Protective role of peroxisome proliferator-activated receptor $\beta / \delta$ in acute lung injury induced by prolonged hyperbaric hyperoxia in rats. Respir Physiol Neurobiol 2014;199:9-18.

24. Chen L, Lan Z. Polydatin attenuates potassium oxonate-induced hyperuricemia and kidney inflammation by inhibiting NF- $\mathrm{B} / \mathrm{NLRP} 3$ inflammasome activation via the AMPK/SIRT1 pathway. Food Funct 2017;8:1785-92.

25. Yu WC, Chen YL, Hwang PA, et al. Fucoidan ameliorates pancreatic $\beta$-cell death and impaired insulin synthesis in streptozotocin-treated $\beta$ cells and mice via a Sirt1-dependent manner. Mol Nutr Food Res 2017. doi: 10.1002/mnfr.201700136.

26. Jia Y, Sun Y, Weng L, et al. Low molecular weight fucoidan protects renal tubular cells from injury induced by albumin overload. Sci Rep 2016;6:31759.

27. Buccellato LJ, Tso M, Akinci OI, et al. Reactive oxygen species are required for hyperoxia-induced Bax activation and cell death in alveolar epithelial cells. J Biol Chem 2004;279:6753-60.

28. Heeba GH, Morsy MA. Fucoidan ameliorates 
steatohepatitis and insulin resistance by suppressing oxidative stress and inflammatory cytokines in experimental non-alcoholic fatty liver disease. Environ Toxicol Pharmacol 2015;40:907-14.

29. Han YS, Lee JH, Jung JS, et al. Fucoidan protects mesenchymal stem cells against oxidative stress and enhances vascular regeneration in a murine hindlimb ischemia model. Int J Cardiol 2015;198:187-95.

30. Yokota T, Nomura K, Nagashima M, et al. Fucoidan alleviates high-fat diet-induced dyslipidemia and atherosclerosis in ApoE(shl) mice deficient in apolipoprotein E expression. J Nutr Biochem 2016;32:46-54.

31. Kondrikov D, Caldwell RB, Dong Z, et al. Reactive oxygen species-dependent RhoA activation mediates collagen synthesis in hyperoxic lung fibrosis. Free Radic

Cite this article as: Zhang Y, Du H, Yu X, Zhu J. Fucoidan attenuates hyperoxia-induced lung injury in newborn rats by mediating lung fibroblasts differentiate into myofibroblasts. Ann Transl Med 2020;8(22):1501. doi: 10.21037/atm-20-6601
Biol Med 2011;50:1689-98.

32. Li J, Chen K, Li S, et al. Protective effect of fucoidan from Fucus vesiculosus on liver fibrosis via the TGF- $\beta 1 / \mathrm{Smad}$ pathway-mediated inhibition of extracellular matrix and autophagy. Drug Des Devel Ther 2016;10:619-30.

33. Chen CH, Sue YM, Cheng CY, et al. Oligo-fucoidan prevents renal tubulointerstitial fibrosis by inhibiting the CD44 signal pathway. Sci Rep 2017;7:40183.

34. Chen J, Cui W, Zhang Q, et al. Low molecular weight fucoidan ameliorates diabetic nephropathy via inhibiting epithelial-mesenchymal transition and fibrotic processes. Am J Transl Res 2015;7:1553-63.

(English Language Editor: J. Chapnick) 